\title{
Critically important antimicrobials are generally not needed to treat nonsevere clinical mastitis in lactating dairy cows: Results from a network meta-analysis
}

\author{
Diego B. Nobrega, ${ }^{1,2 *}$ S. Ali Naqvi, ${ }^{2,3 *}$ Simon Dufour, ${ }^{2,4}$ Rob Deardon, ${ }^{1,5}$ John P. Kastelic, ${ }^{1}$ Jeroen De Buck, ${ }^{1,2}$ \\ and Herman W. Barkema ${ }^{1,2,3} \dagger$ \\ ${ }^{1}$ Department of Production Animal Health, Faculty of Veterinary Medicine, University of Calgary, Calgary, AB, T2N 1N4, Canada \\ ${ }^{2}$ Mastitis Network, Faculty of Veterinary Medicine, University of Montreal, St-Hyacinthe, QC, J2S 7C6, Canada \\ ${ }^{3}$ Department of Community Health Sciences, Cumming School of Medicine, University of Calgary, Calgary, AB, T2N 1N4, Canada \\ ${ }^{4}$ Department of Pathology and Microbiology, Faculty of Veterinary Medicine, University of Montreal, Saint-Hyacinthe, QC, J2S 7C6, Canada \\ ${ }^{5}$ Department of Mathematics and Statistics, Faculty of Science, University of Calgary, Calgary, AB, T2N 1N4, Canada
}

\section{ABSTRACT}

There is ongoing debate regarding whether critically important antimicrobials (CIA) should be used to treat infections in food-producing animals. In this systematic review, we determined whether CIA and non-CIA have comparable efficacy to treat nonsevere bovine clinical mastitis caused by the most commonly reported bacteria that cause mastitis worldwide. We screened CAB Abstracts, Web of Science, MEDLINE, Scopus, and PubMed for original epidemiological studies that assessed pathogen-specific bacteriological cure rates of antimicrobials used to treat nonsevere clinical mastitis in lactating dairy cows. Network models were fit using risk ratios of bacteriological cure as outcome. A total of 30 studies met inclusion criteria. Comparisons of cure rates demonstrated that CIA and non-CIA had comparable efficacy for treatment of nonsevere clinical mastitis in dairy cattle. Additionally, for cows with nonsevere clinical mastitis caused by Escherichia coli and Klebsiella spp., bacteriological cure rates were comparable for treated versus untreated cows; therefore, there was no evidence to justify treatment of these cases with CIA. Our findings supported that CIA in general are not necessary for treating nonsevere clinical mastitis in dairy cattle, the disease that accounts for the majority of antimicrobial usage in dairy herds worldwide. Furthermore, our findings support initiatives to reduce or eliminate use of CIA in dairy herds. Key words: critically important antimicrobial, dairy cow, mastitis, treatment

Received February 13, 2020.

Accepted June 24, 2020.

*These authors contributed equally to this work.

†Corresponding author: barkema@ucalgary.ca

\section{INTRODUCTION}

Antimicrobial resistance is one of the most important global threats to human and animal health. It is estimated that without urgent action, we are heading toward a postantibiotic era where 10 million deaths per year globally will be attributable to antimicrobial resistance (O'Neill, 2016). It is recognized that antimicrobial use contributes to emergence of antimicrobial resistance (Chantziaras et al., 2014); therefore, antimicrobial use should be refined. The World Health Organization (WHO) has promulgated a set of strategies to combat antimicrobial resistance, including reduction of antimicrobial use in food-producing animals.

The WHO classifies antimicrobials into categories based on availability of alternatives and risk of antimicrobial resistance-emergence due to nonhuman antimicrobial use (WHO, 2016). Critically important antimicrobials (CIA) are those used to treat specific diseases in humans, including infections acquired from nonhuman sources. The use of CIA in food-producing animals may be associated with increased risk of nontreatable human infections (Dutil et al., 2010). Hence, there is an ongoing debate on whether these antimicrobials should be used to treat infections in food-producing animals (Collignon, 2013). In the absence of definitive answers, several countries have directed substantial efforts to control overall use of CIA in food-producing animals, either by promoting antimicrobial stewardship or by restricting their use (Dupont et al., 2017). Ideally, alternatives of comparable or superior efficacy should be available as part of any stewardship initiative to reduce use of CIA in food-producing animals. Such alternatives would allow for effective treatment of bacterial infections that negatively affect animal health and welfare, while minimizing their effect on efficacy of CIA for human medicine. 
It is unclear whether CIA are indispensable for treating infections in food-producing animals; an evidencebased assessment would rely on comparisons of efficacy of CIA versus non-CIA. Mastitis accounts for most antimicrobial usage, including CIA, in dairy cattle (Nobrega et al., 2017). Despite numerous randomized clinical trials comparing efficacy of various antimicrobials for treating mastitis (Schukken et al., 2013), approximately all studies assessed only 1 or 2 antimicrobial treatment protocols or antimicrobial classes, thereby falling short to assess the need of CIA to treat mastitis. However, network meta-analysis, a natural extension of meta-analysis (Tonin et al., 2017), efficiently handles multiple treatment protocols and facilitates comparison of results from multiple trials in a single analysis by integrating direct and indirect evidence (e.g., indirect comparison of distinct interventions compared with a same control condition in separate studies).

In this systematic review, we assessed whether CIA and non-CIA have comparable efficacy to treat nonsevere bovine clinical mastitis (CM) caused by the most commonly isolated bacterial pathogens worldwide. We used a set of networks to analyze literature reporting on bacteriological cure rates of antimicrobials used to treat lactating dairy cows with nonsevere CM.

\section{MATERIALS AND METHODS}

\section{Eligibility Criteria}

This systematic review was conducted in accordance with the Preferred Reporting Items for Systematic Reviews and Meta-Analyses (PRISMA) network metaanalysis reporting standards (Hutton et al., 2015). We did not publish the following review protocol before conducting the study. Epidemiological studies (observational or experimental) that assessed pathogen-specific bacteriological cure rates of antimicrobials for treating nonsevere $\mathrm{CM}$ in lactating dairy cows were eligible for inclusion. Nonsevere CM was defined as signs of inflammation in the mammary gland or altered milk secretion with no signs of systemic involvement. The CM cases without any mention of location and severity of inflammation signs were considered nonsevere because that is the most common presentation of $\mathrm{CM}$ in dairy herds (Swinkels et al., 2013b; Kalmus et al., 2014). Intervention was defined as administration of antimicrobials, either systemically or locally (intramammarily). We required inclusion of a comparator group defined as follows: (1) animals with nonsevere CM caused by same pathogen or pathogen group and (2) animals under a different antimicrobial treatment protocol (e.g., different molecule, dose, route of administration, or days under treatment) or untreated. Animals were considered untreated if they received no therapy of any kind or treated with placebo. Consequently, dairy cows that were frequently milked, or that received oxytocin or nonsteroidal anti-inflammatory drugs (NSAID) solely, were not considered untreated. Furthermore, studies evaluating alternative therapies (e.g., frequent milking, NSAID, homeopathy) in addition to antimicrobial treatments were excluded in absence of a second group with a distinct antimicrobial treatment protocol or an untreated group. Studies where antimicrobial treatments were performed following antimicrobial susceptibility testing were not considered unless there were at least 2 treatment protocols in same population stratified by resistance phenotype status (e.g., cows with $\mathrm{CM}$ caused by resistant $S$. aureus under 2 distinct treatment protocols). Bacteriological cure, defined as failure to isolate or detect the same species detected before treatment onset, was considered as the outcome. It was measured at the cow- or quarter-level, outside the withdrawal period but within the first month after last treatment day, using either single or multiple milk samplings. Thus, studies reporting exclusively on clinical cure or other metrics of cure (e.g., improvement, any pathogen cure) were not considered. Milk samples collected within an interval (e.g., 19-41 d) were assumed as collected at the window mean (e.g., $30 \mathrm{~d}$ for the 19-41-d window). Finally, studies only reporting cure rates without any mention of etiological agent were not eligible for inclusion.

\section{Search Strategy and Study Selection}

We screened CAB Abstracts, Web of Science (all databases), MEDLINE, Scopus (Elsevier), and PubMed on January 30, 2019 for potentially relevant articles. We developed a search strategy consisting of relevant key words describing the 3 following themes: population, intervention, and outcome. The broad themes were combined into a single query. Although we did not place a limit on publication date and language during our initial screening, English, Spanish, Portuguese, French, and Italian articles published from 1980 onwards were considered for full-text reviewing. The initial search strategy was designed to be fairly broad. Preliminary assessment of returned hits indicated a substantial number of studies in species other than dairy cattle. Additionally, review articles, case reports, and case series where no comparator was used were also retained by our initial search strategy. Therefore, our search strategy was further refined to exclude review articles and case reports in addition to noncattle studies using the Boolean operator "NOT" at title level 
(Supplemental Text S1, https://doi.org/10.3168/jds .2020-18365). Queries were adapted to database-specific terms, as necessary.

Our search strategy was enhanced by screening relevant conference proceedings using the following 3 specialized databases: (1) Searchable Proceedings of Animal Conferences (SPAC, 2019), (2) National Mastitis Council Proceedings Library (NMC, 2019), and (3) International Veterinary Information Service (IVIS, 2019). The query "mastitis treatment" was used in all 3 databases, and titles from all hits were reviewed. Additionally, abstracts from the third International Mastitis Seminar (IDF et al., 1995; Tel Aviv, Israel) were manually reviewed. Finally, reference lists from all articles included in this systematic review were reviewed for potential inclusion.

Two authors (D. N. and S. A. N.) reviewed all titles and abstracts independently. Original research that reported on efficacy of antimicrobials to treat dairy cows with mastitis during lactation were retained. This initial screening was fairly broad to encompass all potentially relevant studies for full-text review. Discrepancies between reviewers were resolved by consulting with a third reviewer (H. B.). Agreement between reviewers was excellent $(\kappa=0.92 ; 95 \%$ CI: $0.85-0.99)$. All full texts were retrieved and assessed for eligibility using a pre-established screening tool. Epidemiological studies that reported on bacteriological cure rates of $\geq 2$ distinct antimicrobial treatment protocols (or 1 protocol and an untreated group) for treating dairy cows with CM during lactation were retained, according to previously stated eligibility criteria.

\section{Data Collection}

The same reviewers (D. N. and S. A. N.) extracted data independently from individual studies using an electronic form in Microsoft Excel (Redmond, WA). Variables extracted, when available, were author, year, country, study design, CM definition, number of cows and quarters with CM, eligibility criteria for animals included in the study, bacteria, bacteria identification method, antimicrobial treatment, route of administration, dose, days under treatment, type of supportive therapy, time of outcome measurement, level of outcome measurement (i.e., cow or quarter), outcome definition, and numbers of cases (cows or quarters) and cures per treatment protocol. Lastly, authors from studies that had missing required information were contacted by email with regards to availability of data.

For studies in which posttreatment samples were collected on multiple days and cure was assessed individually for each sampling, results from sampling closest to median time of outcome measurement for $\mathrm{CM}$ caused by the same pathogen were selected for extraction. If studies reported nonequivalent cure rates at quarterand cow-level, only the former were retained.

\section{Risk of Bias and Study Quality}

The same 2 reviewers independently assessed quality and risk of bias in individual experimental studies using the Systematic Review Centre for Laboratory Animal Experimentation (SYRCLE) tool (Hooijmans et al., 2014). Risk of bias was classified according to number of error sources that each study had failed to address: low risk of bias (1-3 sources of error), moderate risk of bias (4-6 sources of error), and high risk of bias (7-9 sources of error). A single experimental study classified as having high risk of bias, as well as nonexperimental studies, had their effect on final estimates assessed using sensitivity analysis.

\section{Data Synthesis and Network Meta-Analyses}

Prior to analyses, categories of antimicrobial treatment protocols, supportive therapy, and pathogens were generated. Antimicrobial treatment protocols were grouped using categories defined a priori based on the WHO fifth revision of Critically Important Antimicrobials for Human Medicine (WHO, 2016) and route of administration (systemic and intramammary). The WHO categories included newer generation (third, fourth, and fifth) cephalosporins, macrolides, quinolones, penicillins (natural), amphenicols, penicillins (anti-staphylococcal), and older generation (first and second) cephalosporins. For combinations containing 1 or more antimicrobial or different classes given by different routes, the prioritization category of each molecule was initially determined and used thereafter to classify protocols as follows: (1) combination containing 1 or more highest-priority antimicrobial class or (2) combination containing 1 or more high-priority antimicrobial class. In the few trials where antimicrobials were administered by more than 1 route, protocols were classified as systemic treatments if 1 or more antimicrobial was administered parenterally; their effect on final estimates was assessed through sensitivity analysis, as described. When studies compared effects of routes of administration using systemic, intramammary, and both routes of administration concurrently, results from individual routes were retained exclusively. Finally, because extended therapies are known to affect the probability of bacteriological cure using some antimicrobials (Oliver et al., 2004), and a wide range of days under treatment with cephalosporins was reported (from 1-8), a cut-off of $4 \mathrm{~d}$ was used to define an extended therapy with cephalosporins. For other antimi- 
crobial classes, no significant variations were detected in terms of days under treatment (Supplemental Table S1, https://doi.org/10.3168/jds.2020-18365).

Despite reported lack of efficacy of nonantimicrobial approaches for treating CM (Francoz et al., 2017), use of supportive therapy may affect efficacy of antimicrobials (Roberson et al., 2004). Within a study, each treatment group had its supportive therapy classified as: (1) no use of supportive therapy, (2) intramammary anti-inflammatories (e.g., formulation containing prednisolone), (3) systemic anti-inflammatories (e.g., NSAID), and (4) frequent milking (with or without oxytocin administration). For studies reporting cure rates from multiple antimicrobial treatment protocols where a subset differed on use of supportive therapies exclusively, results from groups receiving supportive therapy were not considered. To be as comprehensive as possible while ensuring a manageable number of treatment categories, use of supportive therapy was handled through sensitivity analyses in network analyses, as described.

Culture results were classified as undetermined (not cultured), Escherichia coli, Klebsiella spp., other coliforms (Enterobacter spp., Citrobacter spp., Serratia spp., or any combination of these bacteria), unspecified coliforms, non-agalactiae streptococci (Streptococcus uberis, Streptococcus dysgalactiae, non-agalactiae streptococci, or any combination of these bacteria), nonaureus staphylococci (coagulase-negative staphylococci, Staphylococcus spp. other than Staphylococcus aureus), S. agalactiae, S. aureus, no growth, and others (Bacillus spp., Pseudomonas spp., Trueperella pyogenes, Nocardia spp., yeasts, molds, Prototheca spp., Enterococcus spp., Corynebacterium spp., Pasteurella spp., Proteus spp.). For any combination of isolates obtained in a single culture, except those described under coliforms and nonagalactiae streptococci, results were classified as mixed culture. However, for studies reporting individual results for each bacterial species, irrespective of presence of a second species in culturing, results from mixed cultures were grouped into 2 pathogen categories, accordingly. Lastly, grouped results excluding those described (e.g., major or minor pathogen, gram-positive or negative) were classified as combined.

Five network meta-analyses were carried out according to the most commonly reported bacteria that cause CM worldwide (S. aureus, E. coli, non-aureus staphylococci, non-agalactiae streptococci, and Klebsiella spp.). Analyses were carried out in $\mathrm{R}$ version 3.5.2 using the "netmeta" package (Rücker et al., 2019). Statistical significance was considered at the $5 \%$ level.

A study was eligible for inclusion in networks if: (1) it reported specific rates of bacteriological cure of nonsevere $\mathrm{CM}$ caused by aforementioned bacteria, (2) it had $\geq 2$ categories of antimicrobial treatments per pathogen after grouping, and (3) 1 or more of these categories was common to $>1$ study included in the pathogenspecific network. If a study provided multiple estimates within the same grouping (e.g., 2 treatment protocols or distinct bacterial species receiving same classification), results were first merged accordingly before assessment of eligibility for inclusion in networks. For within-study pairwise comparisons where no cure was reported for both protocols (probability of cure equal to 0 for 2 protocols within a study), results from the most uncommon protocol were dropped from the analyses. Studies not eligible for inclusion in any network had their results discussed qualitatively.

A frequentist approach using generalized linear models applied using graph theory was used to develop networks (Rücker, 2012). All treatment networks analyzed were closed, as our eligibility criteria for inclusion of a study in networks required common treatment arms between studies. Models were fit using risk ratios for bacteriological cure as the outcome, and random effects to allow for unmeasured, between-study differences. Network inconsistency (a measure of agreement between direct and indirect evidence comparing the same treatments) was assessed using the Q statistic, a measure of discrepancy between the observed risk ratios for a given comparison, compared with what is expected based on any indirect comparisons (Rücker, 2012). Inconsistency was also visually assessed to identify particular studies or comparisons contributing to the inconsistency based on heatmaps generated using a function within "netmeta" (Rücker et al., 2019). The proportion of indirect to direct evidence was also assessed in each model, providing an indication of how many uncommon treatments (present in only a single study) were evaluated and combined with confidence intervals (CI) to assess strength and validity of the findings. When inconsistency was detected, a sensitivity analysis (described below) was conducted to determine the effect of the study's contribution to inconsistency on the overall model fit. Studies removed from the quantitative network meta-analysis were then described qualitatively. Model heterogeneity was assessed using the $\mathrm{I}^{2}$ statistic computed with the random-effects model in "netmeta" (Rücker et al., 2019), representing the ratio of betweenstudy variance to within-study variance. A significant $\mathrm{I}^{2}$ value indicated that unmeasured study characteristics contributed to observed differences more than the treatments themselves. We evaluated all pairwise comparisons of CIA versus each non-CIA using league tables. A CIA category was deemed to be different from non-CIA if relative risks and respective $95 \%$ CI comparing bacteriological cure rates between categories did not include the null value for each pairwise comparison. 
Sensitivity analyses were used to evaluate effects of categories of supportive therapy in our estimates. Treatment protocols containing a specific type of supportive therapy were removed 1 -at-a-time, 2 -at-a-time (intramammary anti-inflammatory and frequent milking exclusively), and all 3 at once. Relative risks and respective $95 \% \mathrm{CI}$, heterogeneity, and inconsistency estimates were compared from a different set of models for each pathogen. Choice of models was based on (1) consistency of findings when compared with models containing only treatment protocols without supportive therapy, (2) lowest heterogeneity (using a threshold of 20 percentage points to infer similarity) and absence of statistical significance for inconsistency estimates, and (3) highest degree of network completeness (defined as the highest possible number of treatment arms or nodes) and studies included while meeting criteria 1 and 2. Finally, a secondary set of sensitivity analyses was carried out 1-at-a-time on selected models to explore potential sources of inconsistencies detected, unusual routes of administration, high risk of bias, nonexperimental evidence, effects of combined route therapies, and pathogen antimicrobial resistance profile. Major findings were robust regardless of presence of refrained studies; choice of models was, therefore, based on heterogeneity and inconsistency estimates using same criteria aforementioned. We did not attempt any additional meta-regression or subgroup analyses for our final models.

A second antimicrobial classification scheme was used to assess the robustness of findings from initial networks. This secondary assessment was done to ensure that very distinct protocols classified as "combinations" in our first assessment did not significantly affect our overall findings, despite low inconsistency and heterogeneity values detected in our initial models. In this secondary scheme, combinations containing one or more antimicrobial were first classified as (1) combination containing aminoglycosides and (2) combination containing anti-staphylococcal penicillins, and analyses were carried out as described. A single study comparing 2 combination protocols that would not fall under the prespecified categories (Sol et al., 2000) was excluded from this secondary assessment. Because findings were robust irrespective of classification scheme, results from initial approach were reported exclusively.

Bias assessment at the reporting level was evaluated using comparison-adjusted funnel plots (Chaimani and Salanti, 2012), where estimated relative risk from random-effects model for specific treatment comparisons were plotted against their respective standard errors. Asymmetry was tested using both Egger's regression test and the Thompson-Sharp regression test, allowing for between-study heterogeneity.

\section{RESULTS}

\section{Description of Studies}

Our search strategy yielded 9,173 records (Figure 1), from which 30 studies were retained (Aguilera, 1983; Guterbock et al., 1993; Lavy et al., 1995; Wilson et al., 1996; Shpigel et al., 1997; McDougall, 1998; Pyorala and Pyorala, 1998; Roberson, 1998; Sol et al., 2000; Hillerton and Kliem, 2002; McDougall, 2003; Taponen et al., 2003a,b; Wraight, 2003; Oliver et al., 2004; Roberson et al., 2004; Sérieys et al., 2005; McDougall et al., 2007a, b; Bradley and Green, 2009; Schukken et al., 2011; Schukken et al., 2013; Swinkels et al., 2013a; Kalmus et al., 2014; Swinkels et al., 2014; Truchetti et al., 2014; Bryan et al., 2016; Cortinhas et al., 2016; Vasquez et al., 2016; Tomazi et al., 2018). Twenty-six studies were included in 1 or more network. Reasons for noninclusion in any network were as follows: (1) flagged as source of inconsistency assessed using the Q statistic and visual inspection of heatmaps (Schukken et al., 2011), (2) high risk of bias (Aguilera, 1983), and (3) absence of $\geq 2$ antimicrobial treatment protocols in different categories following treatment classification (Lavy et al., 1995; McDougall, 2003).

Out of 30 studies, 25 were randomized clinical trials (Table 1). The majority of studies defined CM by using signs of local inflammation and characteristics of milk secretion. Total number of cows enrolled per study ranged from 23 to 1,462. Most studies (28 of 30) reported quarter-level or equivalent cow- and quarterlevel results (e.g., enrollment of single quarter per cow). Only 3 studies used molecular diagnostic techniques for identification of bacteria that cause CM (McDougall et al., 2007a; Schukken et al., 2011; Kalmus et al., 2014).

Most treatment protocols relied on use of 1 or more intramammary antimicrobials (Table 2); number of treatment protocols within a study ranged from 2 to 7 . The CIA of high priority were the most frequently used antimicrobials. Among non-CIA, protocols using first and second generation cephalosporins and anti-staphylococcal penicillins were most often implemented. The most frequently studied pathogen was $S$. aureus $(22$ studies), whereas relatively few $(\mathrm{n}=8)$ studies reported on bacteriological cure of Klebsiella spp.

\section{Escherichia coli}

Fourteen studies reporting on bacteriological cure rates of antimicrobials used to treat nonsevere $\mathrm{CM}$ caused by $E$. coli were eligible for inclusion in networks. From this total, 1 (Schukken et al., 2011) was flagged as a potential source of inconsistency and therefore excluded (Supplemental Table S1, https://doi.org/10 


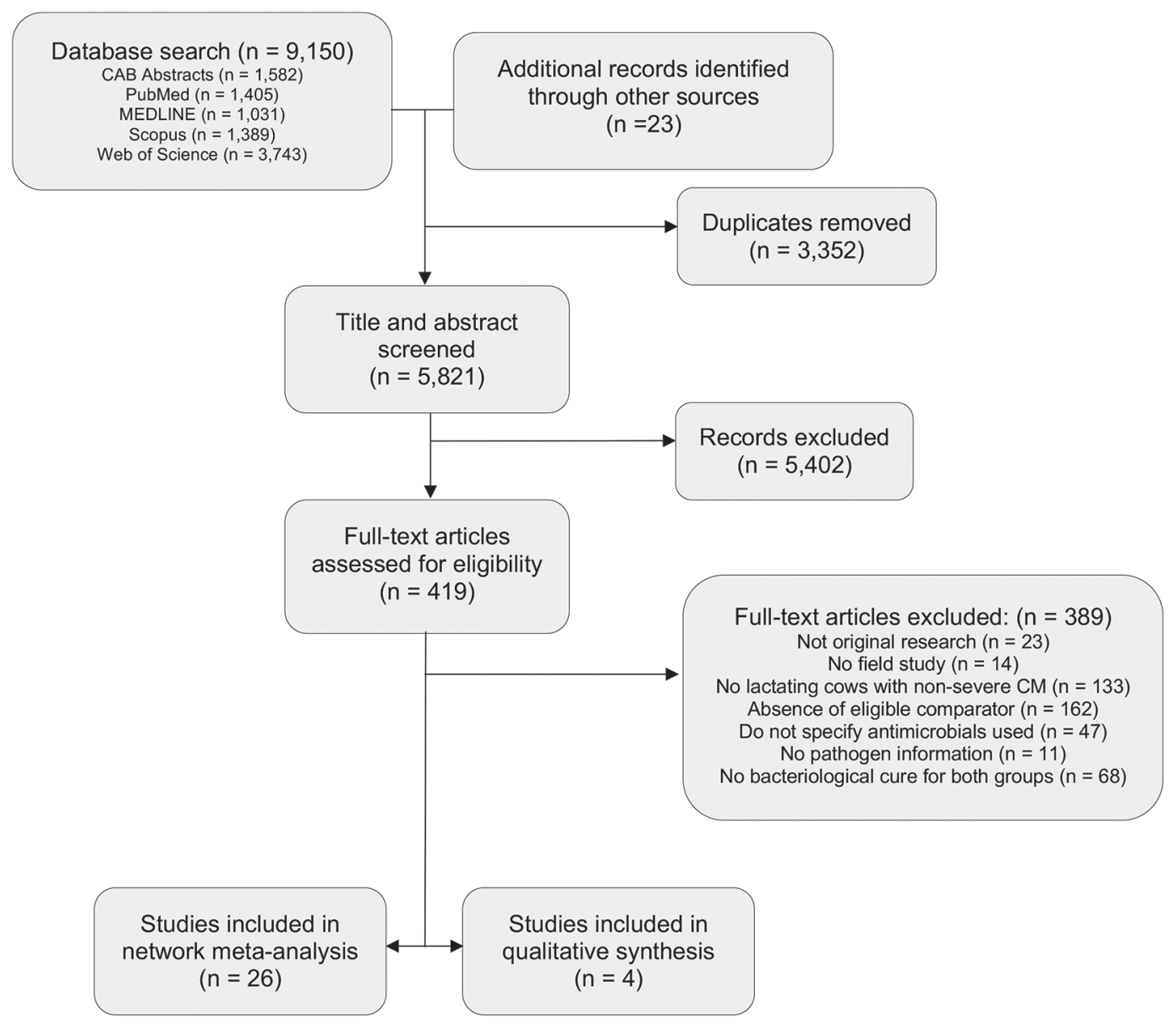

Figure 1. Flow diagram of the study selection process.

.3168/jds.2020-18365). Nevertheless, findings were consistent, irrespective of inclusion of this study; there was no evidence supporting the need of CIA for treating nonsevere CM caused by E. coli (Figure 2). No protocol including the use of CIA had superior bacteriological cure rates of nonsevere $E$. coli $\mathrm{CM}$ than protocols relying on non-CIA (Supplemental Table S2, https://doi .org/10.3168/jds.2020-18365). Additionally, there was no evidence to support use of antimicrobials for treating nonsevere CM caused by E. coli, as the probability of bacteriological cure was similar for treated versus untreated cows (Figure 2).

\section{Klebsiella spp.}

From the 8 studies reporting on bacteriological cure rates of antimicrobials for treating nonsevere $\mathrm{CM}$ caused by Klebsiella spp., 7 were eligible for inclusion in networks. From this total, a single study (Schukken et al., 2011) was excluded because it was a significant source of heterogeneity and inconsistency (Supplemental Table S3, https://doi.org/10.3168/jds.2020-18365). There was no evidence supporting the need to use CIA for treating nonsevere CM caused by Klebsiella spp. (Figure 3, Supplemental Table S4, https://doi.org/10 $.3168 /$ jds.2020-18365), despite inclusion of the potential source of inconsistency in analysis: no protocol including the use of CIA for treatment of nonsevere CM caused by Klebsiella spp. resulted in increased bacteriological cure compared with protocols including non-CIA (Supplemental Table S4). Extended treatment with third generation cephalosporins, antimicrobials classified as CIA, was not superior to protocols based on use of first generation cephalosporins, a non-CIA antimicrobial (relative risk $=1.67,95 \%$ CI: 0.87-3.20; Supplemental Table S4). In addition, probability of bacteriological cure was not lower when no antimicrobials were administered (Figure 3), as reported from 2 trials. Nevertheless, the relatively low sample size of studies involved warrants further investigation. 
Nobrega et al.: CRITICALLY IMPORTANT ANTIMICROBIALS AND MASTITIS

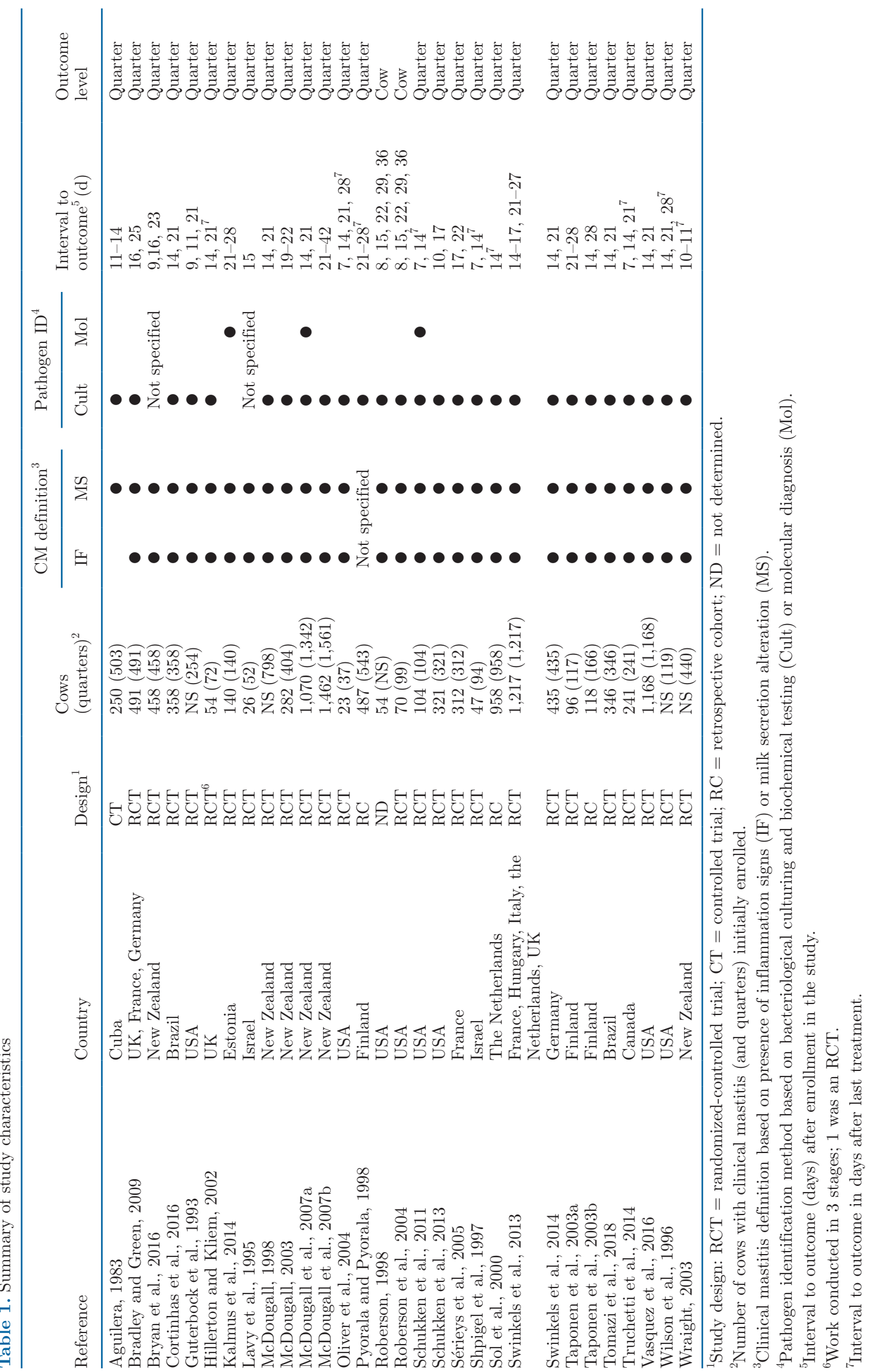




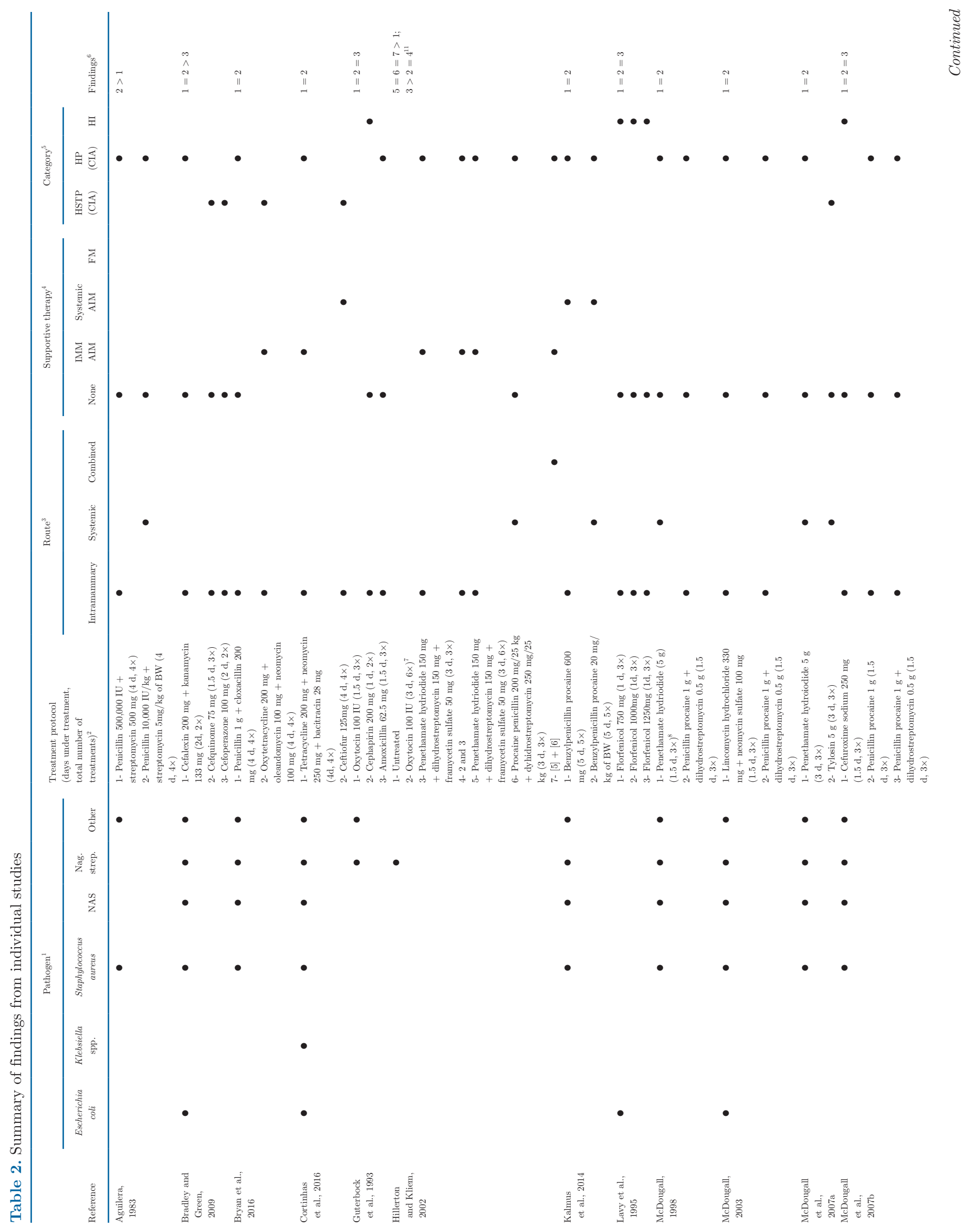

Journal of Dairy Science Vol. 103 No. 11, 2020 


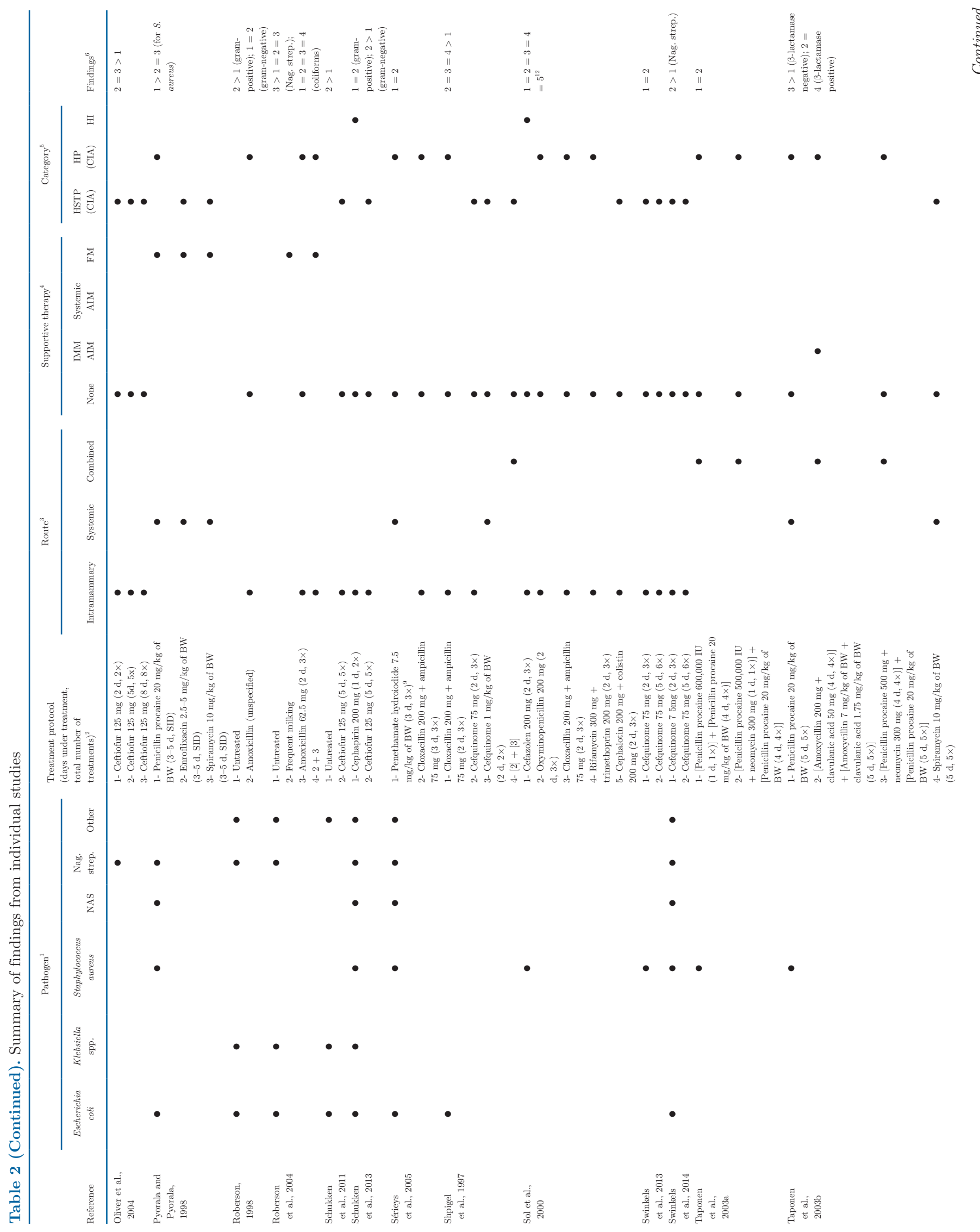

Journal of Dairy Science Vol. 103 No. 11, 2020 


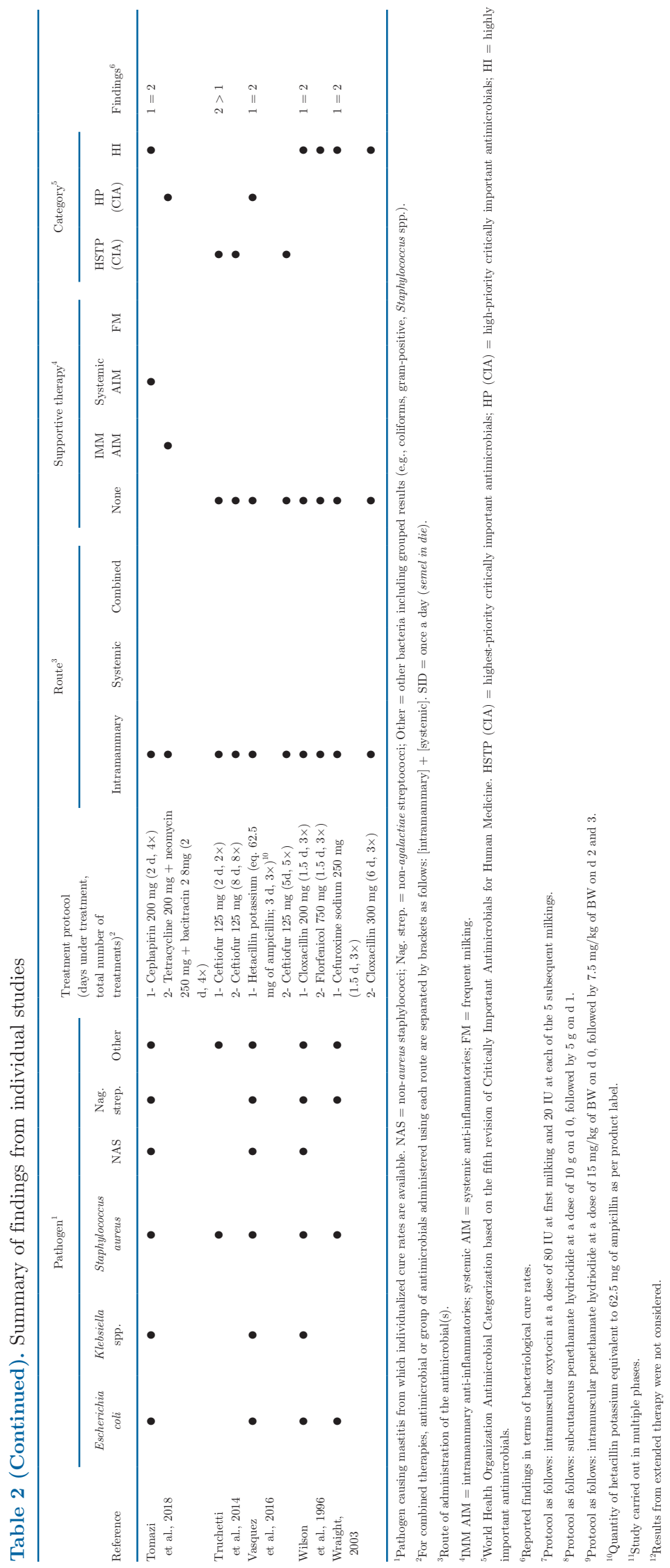




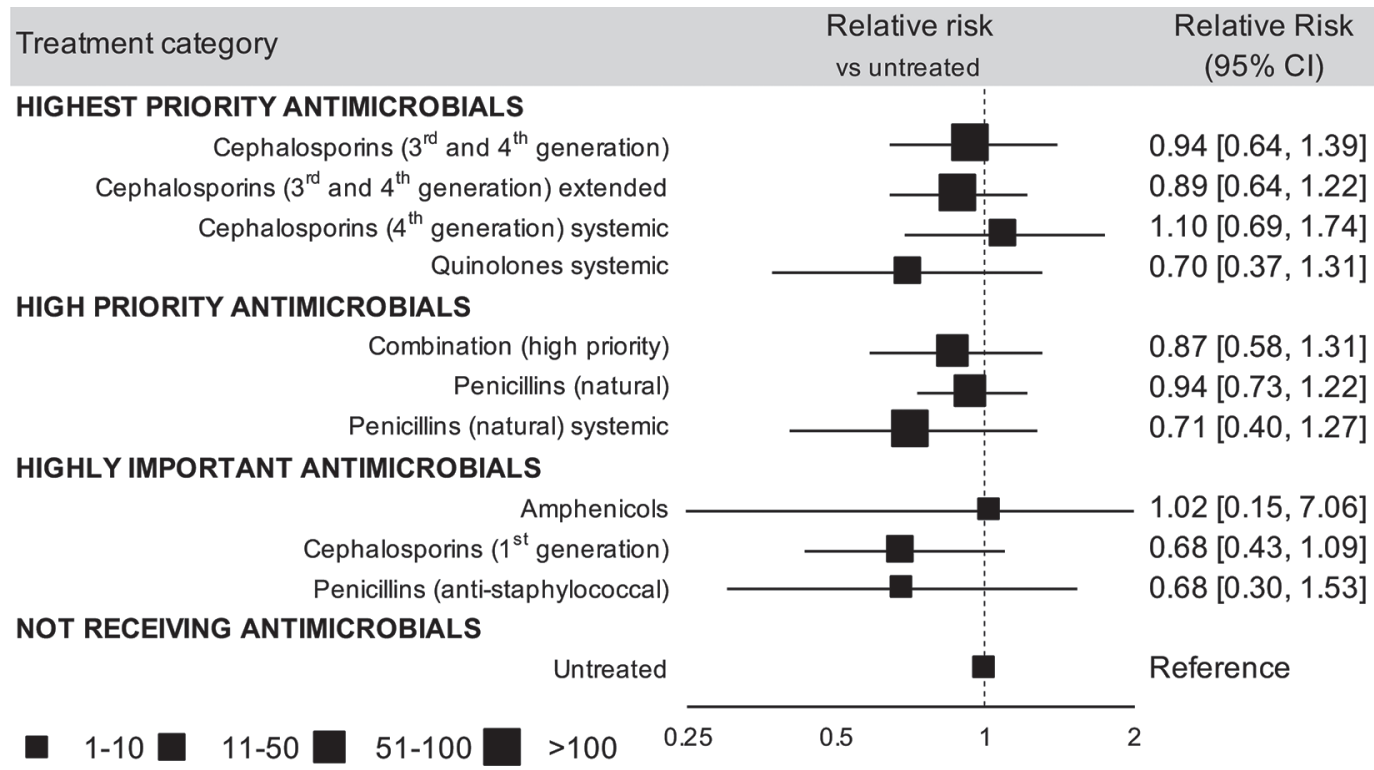

Figure 2. Forest plot of relative risks (and respective $95 \%$ CI) of bacteriological cure of nonsevere bovine clinical mastitis caused by Escherichia coli. Untreated cows were considered as the reference group. Marker size is related to number of units (cows or quarters) enrolled per treatment category after the World Health Organization categorization of medically important antimicrobials.

\section{Non-Aureus Staphylococci}

Fifteen studies reported on bacteriological cure rates of antimicrobials used to treat nonsevere CM caused by non-aureus staphylococci. From this total, 13 were eligible for inclusion in networks, wherein relatively high heterogeneity values were detected in comparison to models for remaining bacterial species (Supplemental Table S5, https://doi.org/10.3168/jds.2020-18365). For treatment of nonsevere CM caused by non-aureus staphylococci, no protocol with use of CIA resulted in increased bacteriological cure when compared with protocols using non-CIA (Figure 4; Supplemental Table S6, https://doi.org/10.3168/jds.2020-18365). Protocols with intramammary administration of third or fourth generation cephalosporins, macrolides or combinations containing CIA of highest priority, were comparable to protocols with intramammary administration of first or second generation cephalosporins for promoting bacteriological cure of nonsevere CM caused by non-aureus staphylococci (Figure 4). No eligible study reported bacteriological cure rates for untreated cows with nonsevere CM caused by nonaureus staphylococci.

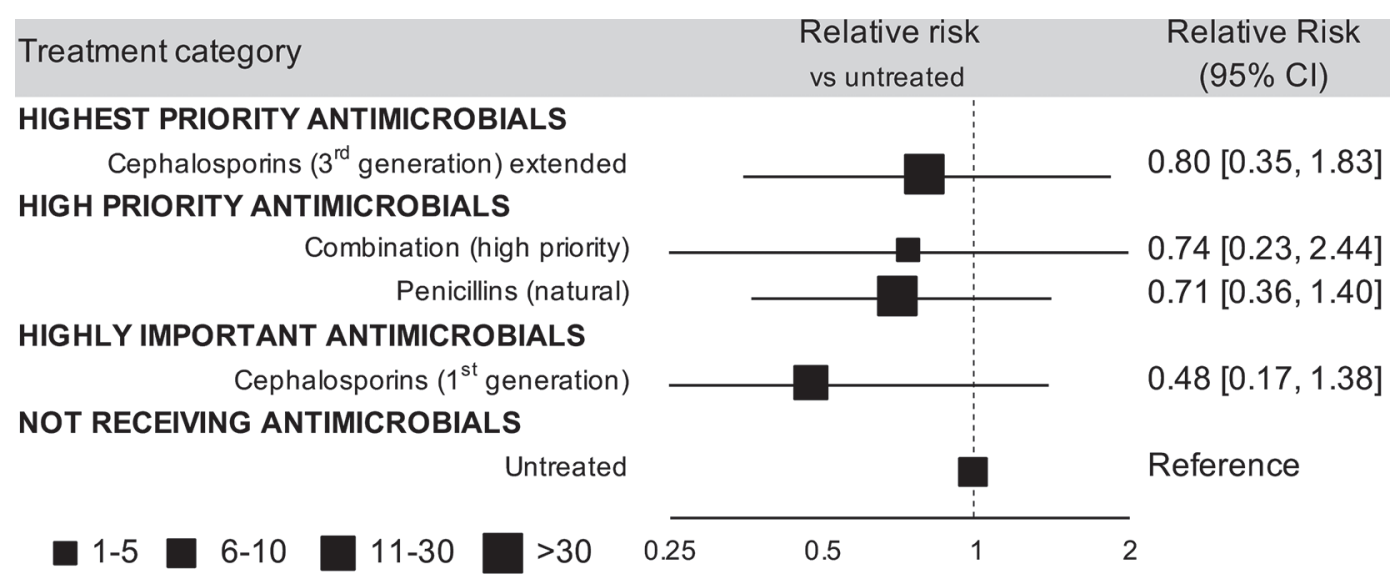

Figure 3. Forest plot of relative risks (and respective $95 \%$ CI) of bacteriological cure of nonsevere bovine clinical mastitis caused by Klebsiella spp. Untreated cows were considered as the reference group. Marker size is related to number of units (cows or quarters) enrolled per treatment category after the World Health Organization categorization of medically important antimicrobials. 


\section{Staphylococcus Aureus}

From the 22 studies reporting on antimicrobial bacteriological cure rates for treatment of nonsevere $\mathrm{CM}$ caused by $S$. aureus, 17 were included in our final $S$. aureus network. Sensitivity analysis indicated that $S$. aureus results depended on inclusion of a study classified as having high risk of bias (Aguilera, 1983), which was therefore excluded. Additionally, our selected $S$. aureus model did not include 4 studies with use of protocols based on intramammary administration of anti-inflammatories due to a marked increase in heterogeneity (Supplemental Table S7, https://doi.org/10 $.3168 /$ jds.2020-18365). The bacteriological cure rate of nonsevere CM caused by $S$. aureus was not different when comparing CIA versus non-CIA; consequently, no CIA category was superior to all non-CIA categories (Supplemental Table S8, https://doi.org/10.3168/jds .2020-18365; Figure 5). No study reporting on spontaneuous bacteriological cure rates of nonsevere $\mathrm{CM}$ caused by $S$. aureus could be included in our networks.

\section{Non-Agalactiae Streptococci}

Twenty-one studies reported on bacteriological cure rates of antimicrobials for treating nonsevere $\mathrm{CM}$ caused by non-agalactiae streptococci, from which 20 were eligible for inclusion in networks. No study or protocol was excluded from networks according to our sensitivity analysis (Supplemental Table S9, https:/ /doi.org/10.3168/jds.2020-18365). No protocol using CIA resulted in higher bacteriological cure rates of nonsevere $\mathrm{CM}$ caused by non-agalactiae streptococci compared with protocols using non-CIA (Supplemental Table S10, https://doi.org/10.3168/jds.2020 -18365). Hence, there was no evidence to support need of CIA for treating nonsevere CM caused by non-agalactiae streptococci (Supplemental Table S10; Figure 6). Nevertheless, administration of antimicrobials was associated with higher probability of bacteriological cure.

\section{Bias Assessments and Network Structures}

Nearly half of the studies used protocols for treatment allocation precluding adequate allocation concealment (Table 3). For most studies, distribution of potential confounders pre-intervention was similar between groups. A potential source of bias common to all studies was housing of cows; it was unclear whether cows from different intervention groups were housed at the same facilities. In general, implementation of blinding at either the performance- or detection-level was infrequent. The most common reason for nonblinded designs was use of very distinct treatment protocols (e.g., 2 vs. 8 d of treatment) in a study. A single study was flagged out as a potential source of significant bias (Table 3) and had its effect on networks evaluated using sensitivity analysis. Additionally, we did not detect evidence of reporting bias across studies (Supplemental Figures S1-S5, https://doi.org/10.3168/jds.2020 -18365). Finally, for all pathogens, network structures revealed a high degree of indirectness, low density, and limited number of direct comparisons of CIA versus non-CIA (Supplemental Figures S6-S10, https://doi .org/10.3168/jds.2020-18365).

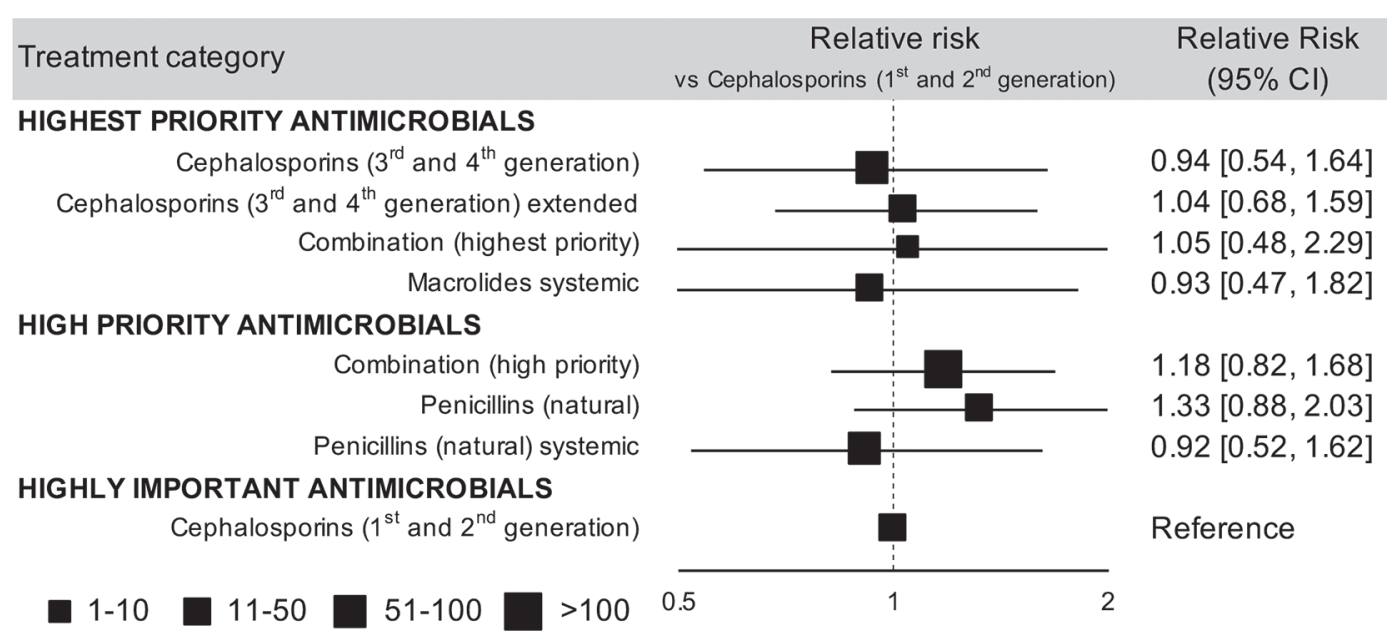

Figure 4. Forest plot of relative risks (and respective 95\% CI) of bacteriological cure of nonsevere bovine clinical mastitis caused by nonaureus staphylococci. Cows receiving intramammary administration of first- or second-generation cephalosporins were considered as the reference group. Marker size is related to number of units (cows or quarters) enrolled per treatment category after the World Health Organization categorization of medically important antimicrobials. 


\section{Qualitative Results}

Two of the 4 studies that could not be included in the network meta-analyses reported on bacteriological cure rates of nonsevere coliform CM (Lavy et al., 1995; Schukken et al., 2011). A positive effect of CIA to treat nonsevere $E$. coli $\mathrm{CM}$ was reported from one trial (Schukken et al., 2011), in contrast with other sources of evidence (Guterbock et al., 1993; Roberson, 1998; Roberson et al., 2004). In addition, no difference between protocols based on increasing dosages of florfenicol (a non-CIA) for treating CM caused by E. coli was reported by the second trial (Lavy et al., 1995). In the 2 remaining trials, gram-positive bacteria were responsible for the majority of CM. Systemic therapy (intra-arterial) with use of high-priority CIA was more effective in treating CM caused by either Streptococcus agalactiae or $S$. aureus than intramammary administration of the same antimicrobials (Aguilera, 1983). Additionally, 2 antimicrobial formulations, both containing high-priority CIA, were equally effective to treat cows with CM infected mostly by $S$. aureus, nonaureus staphylococci, and non-agalactiae streptococci (McDougall, 2003).

\section{DISCUSSION}

To our knowledge, this is the first systematic review comparing efficacy of CIA and non-CIA to treat nonse- vere $\mathrm{CM}$ caused by the most commonly isolated bovine mastitis pathogens worldwide. Findings from this study are important to inform public strategies aimed to promote antimicrobial stewardship in veterinary medicine.

There is an urgent need to restrict and control use of antimicrobials in livestock due to potential adverse effects on human health (Tang et al., 2017). Veterinarians and farmers should choose antimicrobial agents based on a combination of factors including efficacy for treating specific pathogens and potential emergence of antimicrobial resistance following therapy. Although prevalence of antimicrobial resistance in dairy herds has not increased in the last 4 decades (Oliver and $\mathrm{Mu}-$ rinda, 2012), use of CIA in food-producing animals has come under scrutiny in recent years (Apostolakos and Piccirillo, 2018) because of potential negative effects on human and environmental health. Veterinarians should choose non-CIA of comparable or superior efficacy for treating infections in animals as part of any antimicrobial stewardship program. We concluded that CIA and non-CIA have comparable efficacy to treat nonsevere CM in dairy cattle caused by the most commonly isolated pathogens that cause mastitis worldwide. Hence, assuming all other variables that affect safety, choice, and use of antimicrobials are held equal, no adverse effects in terms of animal health and welfare should be expected by ceasing use of CIA for treating nonsevere $\mathrm{CM}$ in herds where no clinical need for CIA had been established (Turner et al., 2018).

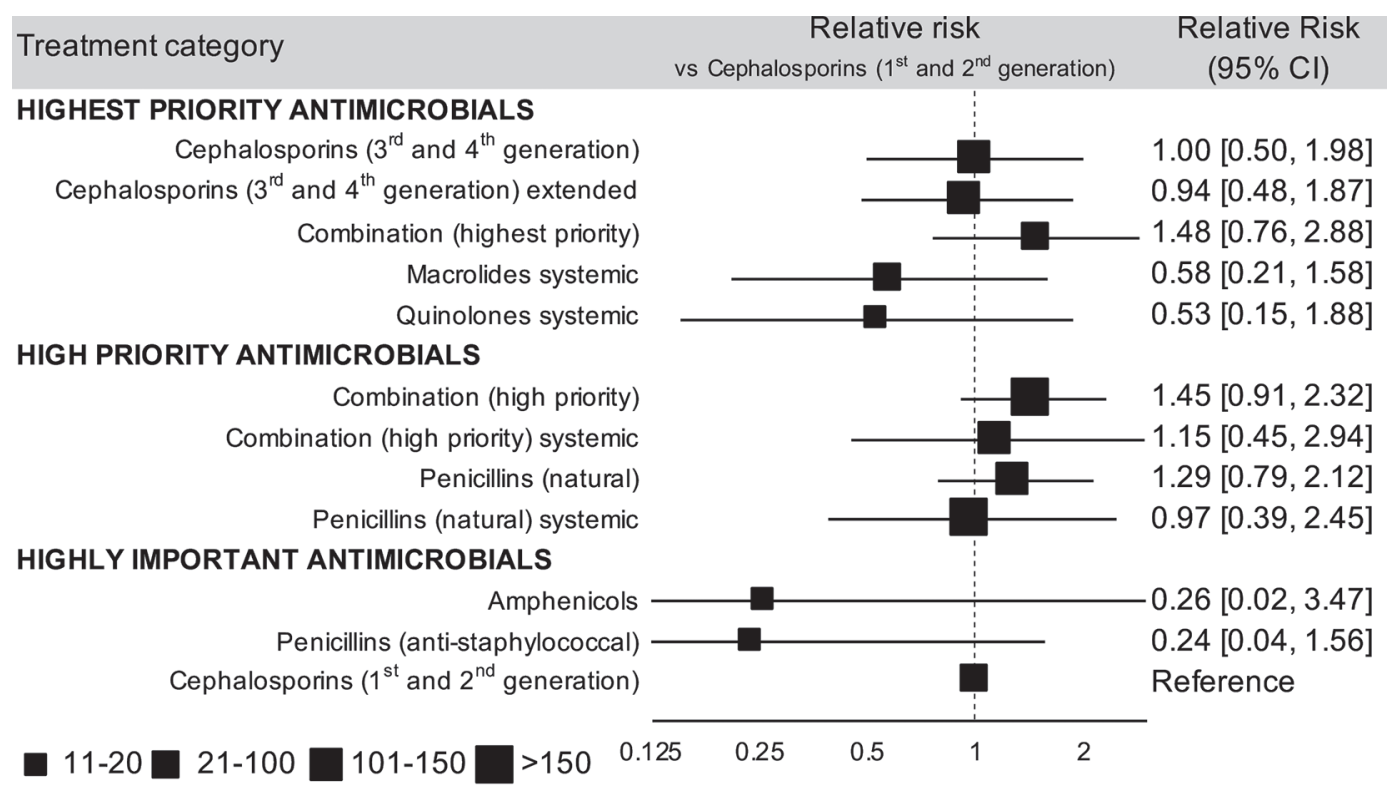

Figure 5. Forest plot of relative risks (and respective 95\% CI) of bacteriological cure of nonsevere bovine clinical mastitis caused by Staphylococcus aureus. Cows receiving intramammary administration of first- or second-generation cephalosporins were considered as the reference group. Marker size is related to number of units (cows or quarters) enrolled per treatment category after the World Health Organization categorization of medically important antimicrobials. 


\begin{tabular}{|c|c|c|}
\hline Treatment category & $\begin{array}{l}\text { Relative risk } \\
\text { vs Cephalosporins ( } 1^{\text {st }} \text { and } 2^{\text {nd }} \text { generation) }\end{array}$ & $\begin{array}{l}\text { Relative Risk } \\
\qquad(95 \% \mathrm{Cl})\end{array}$ \\
\hline \multicolumn{3}{|l|}{ HIGHEST PRIORITY ANTIMICROBIALS } \\
\hline Cephalosporins $\left(3^{\text {rd }}\right.$ and $4^{\text {th }}$ generation $)$ & & $0.95[0.78,1.17]$ \\
\hline Cephalosporins ( $3^{\text {rd }}$ and $4^{\text {th }}$ generation) extended & & $1.11[0.93,1.32]$ \\
\hline Combination (highest priority) & & $0.97[0.77,1.22]$ \\
\hline Macrolides systemic & & $1.01[0.82,1.25]$ \\
\hline \multicolumn{3}{|l|}{ HIGH PRIORITY ANTIMICROBIALS } \\
\hline Combination (high priority) & & $0.97[0.86,1.09]$ \\
\hline Penicillins (natural) & & $0.91[0.80,1.03]$ \\
\hline Penicillins (natural) systemic & & $0.95[0.80,1.13]$ \\
\hline \multicolumn{3}{|l|}{ HIGHLY IMPORTANT ANTIMICROBIALS } \\
\hline Amphenicols & & $-1.79[0.48,6.64]$ \\
\hline Penicillins (anti-staphylococcal) & & $0.90[0.65,1.23]$ \\
\hline Cephalosporins ( $1^{\text {st }}$ and $2^{\text {nd }}$ generation) & & Reference \\
\hline \multicolumn{3}{|l|}{ NOT RECEIVING ANTIMICROBIALS } \\
\hline Untreated & & $0.31[0.13,0.77]$ \\
\hline$\square 1-10 \square 11-50$ & .25 & 2 \\
\hline
\end{tabular}

Figure 6. Forest plot of relative risks (and respective 95\% CI) of bacteriological cure of nonsevere bovine clinical mastitis caused by nonagalactiae streptococci. Cows receiving intramammary administration of first- or second-generation cephalosporins were considered as the reference group. Marker size is related to number of units (cows or quarters) enrolled per treatment category after the World Health Organization categorization of medically important antimicrobials.

Spontaneous cure of intramammary infections and CM caused by gram-positive bacteria is unlikely (Hillerton and Kliem, 2002; Roy et al., 2009); however, the same is not true for CM caused by gram-negative bacteria (Fairbrother et al., 2015). Our findings suggest that antimicrobial treatment is not necessary for nonsevere cases of CM caused by E. coli and Klebsiella spp. Whereas our findings were in agreement with previous studies on bacteriological cure rates of nonsevere CM caused by E. coli (Roberson et al., 2004; Ruegg, 2018; Fuenzalida and Ruegg, 2019), recent evidence demonstrates that antimicrobial therapy of nonsevere CM caused by Klebsiella pneumoniae is associated with higher bacteriological cure rates of treated cows (Fuenzalida and Ruegg, 2019). Unfortunately, included studies reporting bacteriological cure rates for nonsevere CM caused by Klebsiella spp. had a relatively low sample size, limiting our power to assess the need of antimicrobials for treating CM caused by this important pathogen. Nevertheless, irrespective of bacteriological cure, most cows with nonsevere CM caused by coliforms are clinically cured within $1 \mathrm{wk}$ of onset of CM, regardless of treatment. In addition, there are no differences in terms of mastitis recurrence, apparent culling rates, and voluntary dry-off between treated and nontreated quarters affected by coliforms (Fuenzalida and Ruegg, 2019), suggesting that empirical antimicrobial treatment of nonsevere CM caused by coliforms using CIA is irresponsible. In that regard, use of culture-based, selective treatment programs where antimicrobials are not used for cases of mastitis caused by gram-negative bacteria can be a valuable alternative. Nonetheless, although our findings supported use of selective treatment of $\mathrm{CM}$, we must emphasize that our main objective was to contrast CIA versus non-CIA for treatment of nonsevere CM.

The WHO list of CIA was developed with regard to the importance of antimicrobials in human medicine; cost was not a primary factor for classifying an antimicrobial as critically important. This classification scheme informs decision-making, particularly strategies for antimicrobial administration in food-producing animals (Collignon et al., 2016). In veterinary epidemiology, decision-making should factor in expected economical outcomes (James, 2005); costs of interventions are expected to directly affect dynamics of animal diseases. Restrictions of antimicrobial use in dairy cattle could increase milk price, decreasing gross revenue for dairy farmers as well as affecting markets (Lhermie et al., 2018). A framework for tackling antimicrobial resistance not only relies on reducing overall antimicrobial consumption, but also on ensuring that the most effective narrow-spectrum antimicrobial is used when necessary (Om et al., 2016). Our findings support policies to reduce or eliminate use of CIA in dairy herds. Yet, despite a palpable sense of urgency due to alarming antimicrobial resistance rates worldwide, we must stress that phasing out use of CIA to treat CM will depend on 
Nobrega et al.: CRITICALLY IMPORTANT ANTIMICROBIALS AND MASTITIS

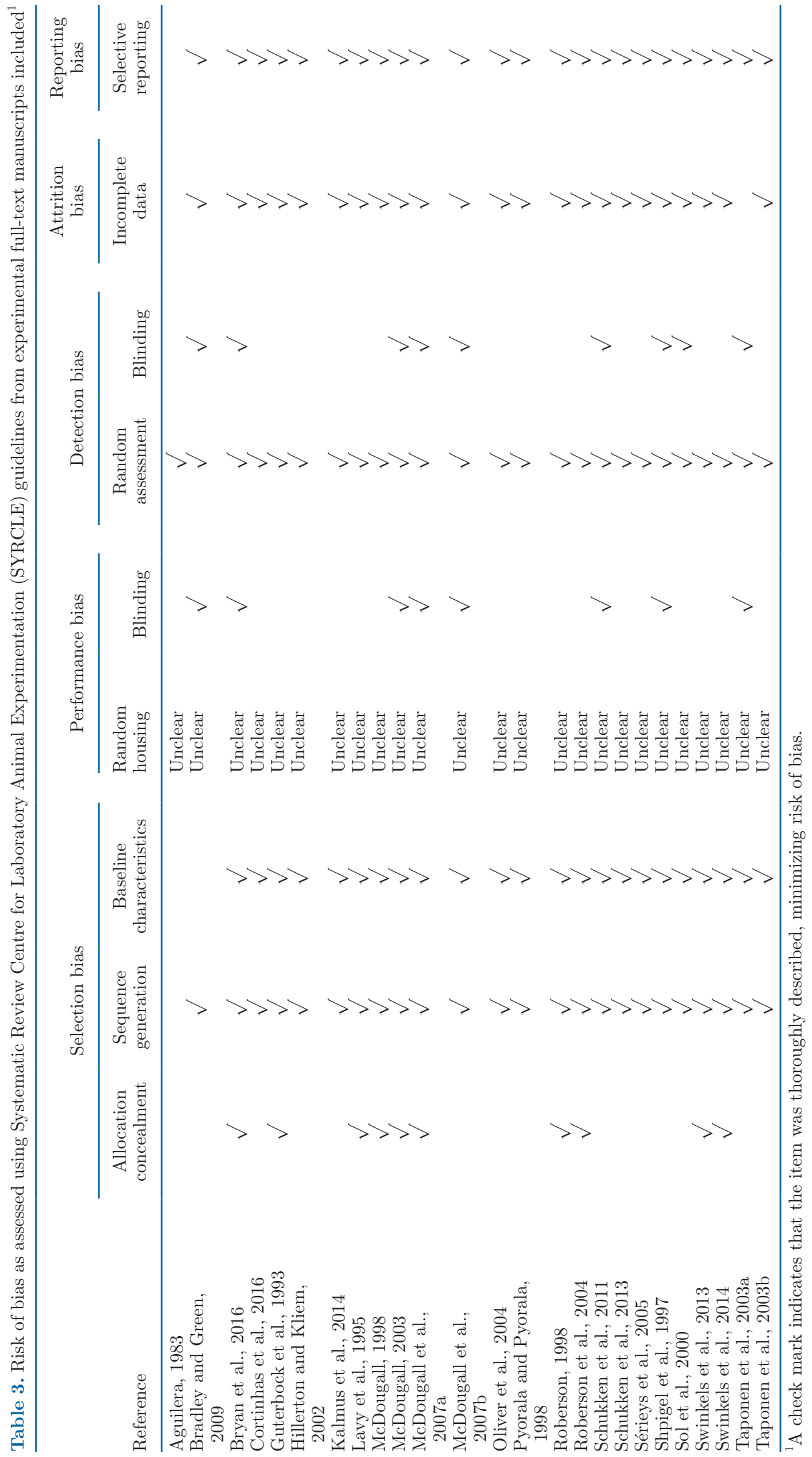


other factors, for example, whether alternatives demonstrate efficacy in cases of intramammary infections caused by bacteria resistant to non-CIA. Additionally, despite comparable efficacy, economic factors such as costs of specific antimicrobials, duration of milk withdrawal, duration of therapy and route of administration must also be included in the equation when selecting an antimicrobial for mastitis treatment. Therefore, our findings should be used as an important part of a discussion for implementing strategies to manage $\mathrm{CM}$ in dairy herds that align with international recommendations for combating antimicrobial resistance.

There is not enough evidence to support exclusive use of supportive therapy (e.g., frequent milking and homeopathy) for treating nonsevere CM in dairy cattle (Morin et al., 1998; Francoz et al., 2017). Use of supportive therapy might even be detrimental to udder health (Roberson et al., 2004). We handled use of supportive therapy in 3 ways: (1) by performing a set of sensitivity analysis, (2) by excluding studies where treatment protocols differed exclusively on use of supportive therapy, and (3) by extracting data from groups not receiving supportive therapy in studies reporting individualized cure rates for $\geq 2$ antimicrobials, with and without supportive therapy. For all pathogens except for $S$. aureus, findings and model estimates were comparable, despite inclusion of protocols with use of supportive therapy. Surprisingly, for S. aureus, exclusion of protocols with use of intramammary anti-inflammatories resulted in a sharp decrease in heterogeneity estimates, suggesting that efficacy of a subset of antimicrobials depended on intramammary administration of anti-inflammatories. It was suggested that bacterins administered in conjunction with pirlimycin might increase probability of bacteriological cure for cows with intramammary infections caused by $S$. aureus (Luby and Middleton, 2005 ), potentially due to reduced inflammation resulting from a focused immune response. Similarly, reduced inflammation due to intramammary administration of anti-inflammatories could potentially affect the overall probability of cure, resulting in increased heterogeneity estimates as detected in our networks.

The goal of antimicrobial therapy is to enhance bacterial clearance; efficacy of products used to treat CM is initially evaluated using estimates of bacteriologic cure rates (Ruegg, 2018). We excluded studies that did not report bacteriological cure rates; our choice of outcome relied on a balance between number of eligible studies and heterogeneity. Ideally, one would use an elaborate metric of cure based on lack of clinical signs and elimination of causative pathogen (e.g., complete cure). Yet, adoption of such a metric would inevitably lead to exclusion of a majority of studies, as this information was not available. Alternatively, a large increase in number of eligible studies can be achieved by inclusion of studies reporting clinical cure. Nonetheless, as mastitis cure rates depend on the causal pathogens (Vasquez et al., 2016), the information provided by clinical cure in absence of bacteriological culturing is potentially a source of heterogeneity when evaluating effects of antimicrobials. If, for instance, treatment assignment depended on underlying causal bacteria in studies reporting exclusively on clinical cure, there would have been increased heterogeneity or inconsistency in our networks. Such discrepancy could arise, for example, from distinct pathogen-specific incidence rates that are commonly reported from countries (Oliveira et al., 2013; 2015).

Some limitations of this study must be acknowledged. First, non-agalactiae streptococci and nonaureus staphylococci are diverse groups. Antimicrobial efficacy depends on the causal pathogen, and it is unclear whether efficacy of a specific antimicrobial will be similar for CM cases caused by different pathogens (e.g., S. uberis versus S. dysgalactiae or S. chromogenes versus $S$. epidermidis). Similarly, in the absence of molecular identification techniques such as MALDI-TOF, non-aureus staphylococci were typically only identified at the group level when based on culture alone. Without differentiating non-aureus staphylococci species, it is difficult to distinguish between a failure to cure and a successful cure followed by a new IMI by another non-aureus staphylococci species. Additionally, network meta-analyses inevitably depend on some degree of grouping. Although we tested 2 antimicrobial categorization schemes, a wide variety of antimicrobial treatment protocols receive the same WHO categorization. Nevertheless, the relatively low heterogeneity and nonsignificant inconsistency estimates were reassuring; not only was the between-study variance for same comparison in a species relatively low, but direct and indirect sources of evidence were in overall agreement. Second, despite nonsignificant inconsistency estimates, the majority of evidence from networks was indirect and should be interpreted accordingly. Of note, the limited number of studies and elevated number of treatment protocols resulted in low-density networks, which was expected due to diversity of treatment protocols for CM available worldwide. Additionally, low heterogeneity detected in the networks was mostly a consequence of lack of common treatment arms across studies. In this scenario, the assumption of transitivity (no systematic differences among comparisons of same treatments) becomes crucial (Salanti et al., 2014). Third, for CM cases caused by Klebsiella spp., and for specific antimicrobial categories (e.g., amphenicols, anti-staphylococcal penicillins), low sample size reduced our probability of detecting meaningful associations; absence of statistical significance at $5 \%$ level in this 
case could have been due to lack of statistical power. Moreover, our study ignored the concept of margin of noninferiority. For bacteriological cure rates differing within a pre-established value, its adoption would ensure that antimicrobials from separate categories were deemed noninferior to each other in terms of overall efficacy, despite presence of statistical significance. Finally, findings were specific for the current scenario in terms of antimicrobial alternatives for treating CM; development of new antimicrobials, formulations, or treatment protocols was not captured by our models. Similarly, CIA and non-CIA included in our analysis did not encompass all possible antimicrobial molecules and classes from these categories. Therefore, we do not recommend extrapolation of our findings to nonincluded antimicrobials or treatment protocols.

\section{CONCLUSIONS}

Our findings support that antimicrobial treatment does not improve the outcome for nonsevere $\mathrm{CM}$ caused by E. coli. Additionally, CIA and non-CIA had comparable efficacy for treating nonsevere CM caused by the 5 most prevalent mastitis-causing pathogens or group of pathogens worldwide $(S$. aureus, non-aureus staphylococci, non-agalactiae streptococci, E. coli, and Klebsiella spp.). Findings from this study support public strategies that promote responsible antimicrobial stewardship in veterinary medicine.

\section{ACKNOWLEDGMENTS}

D. B. N. received funding and support from an NSERC CREATE in milk quality graduate scholarship (Saint-Hyacinthe, QC, Canada), Alberta Innovates Technology Futures scholarship (Alberta, Canada), and the Izaak Walton Killam Memorial Scholarship (Calgary, Canada), with additional funding from the Gustav Rosenberger Memorial Grant. S. A. N. received additional funding and support from an NSERC CREATE in milk quality graduate scholarship and the Workforce Development Initiative graduate student scholarship from the Canadian Dairy Network (Guelph, ON, Canada). The authors have not stated any conflicts of interest.

\section{REFERENCES}

Aguilera, R. 1983. Influence of the course of mastitis and the type of mammary secretion on the effectiveness of treatment with strepto penicillin by different routes. Rev. Salud Anim. 5:1-14.

Apostolakos, I., and A. Piccirillo. 2018. A review on the current situation and challenges of colistin resistance in poultry production. Avian Pathol. 47:546-558. https://doi.org/10.1080/03079457.2018 .1524573 .
Bradley, A. J., and M. J. Green. 2009. Factors affecting cure when treating bovine clinical mastitis with cephalosporin-based intramammary preparations. J. Dairy Sci. 92:1941-1953. https://doi.org/ 10.3168/jds.2008-1497.

Bryan, M. A., S. Y. Hea, S. A. Mannering, and R. Booker. 2016. Demonstration of non-inferiority of a novel combination intramammary antimicrobial in the treatment of clinical mastitis. N. Z. Vet. J. 64:337-342. https://doi.org/10.1080/00480169.2016.1210044.

Chaimani, A., and G. Salanti. 2012. Using network meta-analysis to evaluate the existence of small-study effects in a network of interventions. Res. Synth. Methods 3:161-176. https://doi.org/10 $.1002 / \mathrm{jrsm} .57$.

Chantziaras, I., F. Boyen, B. Callens, and J. Dewulf. 2014. Correlation between veterinary antimicrobial use and antimicrobial resistance in food-producing animals: A report on seven countries. J. Antimicrob. Chemother. 69:827-834. https://doi.org/10.1093/jac/ dkt443.

Collignon, P. 2013. Ban routine use of critically important antibiotics in food animals. BMJ 347:f4976. https://doi.org/10.1136/bmj .f4976.

Collignon, P. J., J. M. Conly, A. Andremont, S. A. McEwen, and A. Aidara-Kane. 2016. World Health Organization ranking of antimicrobials according to their importance in human medicine: A critical step for developing risk management strategies to control antimicrobial resistance from food animal production. Clin. Infect. Dis. 63:1087-1093. https://doi.org/10.1093/cid/ciw475.

Cortinhas, C. S., T. Tomazi, M. S. F. Zoni, E. Moro, and M. Veiga dos Santos. 2016. Randomized clinical trial comparing ceftiofur hydrochloride with a positive control protocol for intramammary treatment of nonsevere clinical mastitis in dairy cows. J. Dairy Sci. 99:5619-5628. https://doi.org/10.3168/jds.2016-10891.

Dupont, N., L. H. Diness, M. Fertner, C. S. Kristensen, and H. Stege. 2017. Antimicrobial reduction measures applied in Danish pig herds following the introduction of the "Yellow Card" antimicrobial scheme. Prev. Vet. Med. 138:9-16. https://doi.org/10.1016/j .prevetmed.2016.12.019.

Dutil, L., R. Irwin, R. Finley, L. K. Ng, B. Avery, P. Boerlin, A. M. Bourgault, L. Cole, D. Daignault, A. Desruisseau, W. Demczuk, L. Hoang, G. B. Horsman, J. Ismail, F. Jamieson, A. Maki, A. Pacagnella, and D. R. Pillai. 2010. Ceftiofur resistance in Salmonella enterica serovar Heidelberg from chicken meat and humans, Canada. Emerg. Infect. Dis. 16:48-54. https://doi.org/10.3201/ eid1601.090729.

Fairbrother, J. H., S. Dufour, J. M. Fairbrother, D. Francoz, E. Nadeau, and S. Messier. 2015. Characterization of persistent and transient Escherichia coli isolates recovered from clinical mastitis episodes in dairy cows. Vet. Microbiol. 176:126-133. https://doi .org/10.1016/j.vetmic.2014.12.025.

Francoz, D., V. Wellemans, J. P. Dupre, J. P. Roy, F. Labelle, P. Lacasse, and S. Dufour. 2017. Invited review: A systematic review and qualitative analysis of treatments other than conventional antimicrobials for clinical mastitis in dairy cows. J. Dairy Sci. 100:77517770. https://doi.org/10.3168/jds.2016-12512.

Fuenzalida, M. J., and P. L. Ruegg. 2019. Negatively controlled, randomized clinical trial to evaluate intramammary treatment of nonsevere, Gram-negative clinical mastitis. J. Dairy Sci. 102:54385457. https://doi.org/10.3168/jds.2018-16156.

Guterbock, W. M., A. L. Vaneenennaam, R. J. Anderson, I. A. Gardner, J. S. Cullor, and C. A. Holmberg. 1993. Efficacy of intramammary antibiotic-therapy for treatment clinical mastitis caused by environmental pathogens. J. Dairy Sci. 76:3437-3444. https://doi .org/10.3168/jds.S0022-0302(93)77682-1.

Hillerton, J. E., and K. E. Kliem. 2002. Effective treatment of Streptococcus uberis clinical mastitis to minimize the use of antibiotics. J. Dairy Sci. 85:1009-1014. https://doi.org/10.3168/jds.S0022 $-0302(02) 74161-1$.

Hooijmans, C. R., M. M. Rovers, R. B. de Vries, M. Leenaars, M. Ritskes-Hoitinga, and M. W. Langendam. 2014. SYRCLE's risk of bias tool for animal studies. BMC Med. Res. Methodol. 14:43. https://doi.org/10.1186/1471-2288-14-43. 
Hutton, B., G. Salanti, D. M. Caldwell, A. Chaimani, C. H. Schmid, C. Cameron, J. P. Ioannidis, S. Straus, K. Thorlund, J. P. Jansen, C. Mulrow, F. Catala-Lopez, P. C. Gotzsche, K. Dickersin, I. Boutron, D. G. Altman, and D. Moher. 2015. The PRISMA extension statement for reporting of systematic reviews incorporating network meta-analyses of health care interventions: checklist and explanations. Ann. Intern. Med. 162:777-784. https://doi.org/10 .7326/M14-2385.

IDF International Mastitis Seminar. A. Saran, S. Soback, IDF Group of Experts Dealing with Bovine Mastitis, and International Dairy Federation. 1995. Proc. 3rd Intern. Mastitis Seminar, Tel Aviv, Israel. National Mastitis Reference Center, Kimron Veterinary Institute, Beit-Dagan, Israel.

International Veterinary Information Service. 2019. https://www.ivis .org/home.asp. Accessed Feb. 9, 2020.

James, A. 2005. The state of veterinary epidemiology and economics. Prev. Vet. Med. 67:91-99. https://doi.org/10.1016/j.prevetmed .2004.11.003

Kalmus, P., H. Simojoki, T. Orro, S. Taponen, K. Mustonen, J. Holopainen, and S. Pyorala. 2014. Efficacy of 5-day parenteral versus intramammary benzylpenicillin for treatment of clinical mastitis caused by gram-positive bacteria susceptible to penicillin in vitro. J. Dairy Sci. 97:2155-2164. https://doi.org/10.3168/jds.2013 -7338 .

Lavy, E., N. Y. Shpigel, G. Ziv, A. Saran, M. Winkler, and K. J. Varma. 1995. Clinical and bacteriological evaluation of florfenicol in the intramammary treatment of experimental $E$. coli mastitis. Pages 23-27 in Proc. 3rd Intern. Mastitis Seminar, Tel Aviv, Israel. National Mastitis Reference Center, Kimron Veterinary Institute, Beit-Dagan, Israel.

Lhermie, G., L. W. Tauer, and Y. T. Grohn. 2018. An assessment of the economic costs to the U.S. dairy market of antimicrobial use restrictions. Prev. Vet. Med. 160:63-67. https://doi.org/10.1016/j .prevetmed.2018.09.028.

Luby, C. D., and J. R. Middleton. 2005. Efficacy of vaccination and antibiotic therapy against Staphylococcus aureus mastitis in dairy cattle. Vet. Rec. 157:89-90. https://doi.org/10.1136/vr.157.3.89.

McDougall, S. 1998. Efficacy of two antibiotic treatments in curing clinical and subclinical mastitis in lactating dairy cows. N. Z. Vet. J. 46:226-232. https://doi.org/10.1080/00480169.1998.36094.

McDougall, S. 2003. Intramammary treatment of clinical mastitis of dairy cows with a combination of lincomycin and neomycin, or penicillin and dihydrostreptomycin. N. Z. Vet. J. 51:111-116. https://doi.org/10.1080/00480169.2003.36349.

McDougall, S., K. E. Agnew, R. Cursons, X. X. Hou, and C. R. W. Compton. 2007a. Parenteral treatment of clinical mastitis with tylosin base or penethamate hydriodide in dairy cattle. J. Dairy Sci. 90:779-789. https://doi.org/10.3168/jds.S0022-0302(07)71562-X.

McDougall, S., D. G. Arthur, M. A. Bryan, J. J. Vermunt, and A. M Weir. 2007b. Clinical and bacteriological response to treatment of clinical mastitis with one of three intramammary antibiotics. N. Z. Vet. J. 55:161-170. https://doi.org/10.1080/00480169.2007.36762.

Morin, D. E., R. D. Shanks, and G. C. McCoy. 1998. Comparison of antibiotic administration in conjunction with supportive measures versus supportive measures alone for treatment of dairy cows with clinical mastitis. J. Am. Vet. Med. Assoc. 213:676-684.

National Mastitis Council. 2019. National Mastitis Council Proceedings Library. Vol. 2018. Accessed Feb. 9, 2020. http://nmconline .omnibooksonline.com/?qr=1.

Nobrega, D. B., J. De Buck, S. A. Naqvi, G. Liu, S. Naushad, V. Saini, and H. W. Barkema. 2017. Comparison of treatment records and inventory of empty drug containers to quantify antimicrobial usage in dairy herds. J. Dairy Sci. 100:9736-9745. https://doi.org/10 $.3168 /$ jds.2017-13116

O'Neill, J. 2016. Tackling drug-resistant infections globally: Final report and recommendations. The review on antimicrobial resistance. Accessed Feb. 9, 2020. https://amr-review.org/sites/default/files/ 160518_Final\%20paper_with\%20cover.pdf.

Oliveira, C. S., H. Hogeveen, A. M. Botelho, P. V. Maia, S. G. Coelho, and J. P. Haddad. 2015. Cow-specific risk factors for clinical mas- titis in Brazilian dairy cattle. Prev. Vet. Med. 121:297-305. https: //doi.org/10.1016/j.prevetmed.2015.08.001.

Oliveira, L., C. Hulland, and P. L. Ruegg. 2013. Characterization of clinical mastitis occurring in cows on 50 large dairy herds in Wisconsin. J. Dairy Sci. 96:7538-7549. https://doi.org/10.3168/jds .2012-6078.

Oliver, S. P., R. A. Almeida, B. E. Gillespie, S. J. Headrick, H. H. Dowlen, D. L. Johnson, K. C. Lamar, S. T. Chester, and W. M. Moseley. 2004. Extended ceftiofur therapy for treatment of experimentally-induced Streptococcus uberis mastitis in lactating dairy cattle. J. Dairy Sci. 87:3322-3329. https://doi.org/10.3168/jds .S0022-0302(04)73468-2.

Oliver, S. P., and S. E. Murinda. 2012. Antimicrobial resistance of mastitis pathogens. Vet. Clin. North Am. Food Anim. Pract. 28:165-185. https://doi.org/10.1016/j.cvfa.2012.03.005.

Om, C., F. Daily, E. Vlieghe, J. C. McLaughlin, and M. L. McLaws. 2016. "If it's a broad spectrum, it can shoot better": Inappropriate antibiotic prescribing in Cambodia. Antimicrob. Resist. Infect. Control 5:58. https://doi.org/10.1186/s13756-016-0159-7.

Pyorala, S. H., and E. O. Pyorala. 1998. Efficacy of parenteral administration of three antimicrobial agents in treatment of clinical mastitis in lactating cows: 487 cases (1989-1995). J. Am. Vet. Med. Assoc. 212:407-412.

Roberson, J. R. 1998. Intramammary (IMM) amoxicillin versus no treatment for cases of subacute clinical mastitis. Pages 282-283 in Proc. 37th Ann. Mtg. National Mastitis Council, St. Louis, MO. National Mastitis Council, New Prague, MN.

Roberson, J. R., L. D. Warnick, and G. Moore. 2004. Mild to moderate clinical mastitis: efficacy of intramammary amoxicillin, frequent milk-out, a combined intramammary amoxicillin, and frequent milk-out treatment versus no treatment. J. Dairy Sci. 87:583-592. https://doi.org/10.3168/jds.S0022-0302(04)73200-2.

Roy, J.-P., L. DesCoteaux, D. DuTremblay, F. Beaudry, and J. Elsener. 2009. Efficacy of a 5-day extended therapy program during lactation with cephapirin sodium in dairy cows chronically infected with Staphylococcus aureus. Can. Vet. J. 50:1257-1262.

Rücker, G. 2012. Network meta-analysis, electrical networks and graph theory. Res. Synth. Methods 3:312-324. https://doi.org/10.1002/ jrsm.1058.

Rücker, G., U. Krahn, J. Konig, O. Efthimiou, and G. Schwarzer. 2019. netmeta: network meta-analysis using frequentist methods. R package version 1.0-1. Accessed Jul. 2020. https://cran.r -project.org/web/packages/netmeta/netmeta.pdf.

Ruegg, P. L. 2018. Making antibiotic treatment decisions for clinical mastitis. Vet. Clin. North Am. Food Anim. Pract. 34:413-425. https://doi.org/10.1016/j.cvfa.2018.06.002.

Salanti, G., C. Del Giovane, A. Chaimani, D. M. Caldwell, and J. P. Higgins. 2014. Evaluating the quality of evidence from a network meta-analysis. PLoS One 9:e99682. https://doi.org/10.1371/ journal.pone.0099682.

Schukken, Y. H., G. J. Bennett, M. J. Zurakowski, H. L. Sharkey, B. J. Rauch, M. J. Thomas, B. Ceglowski, R. L. Saltman, N. Belomestnykh, and R. N. Zadoks. 2011. Randomized clinical trial to evaluate the efficacy of a 5-day ceftiofur hydrochloride intramammary treatment on nonsevere gram-negative clinical mastitis. J. Dairy Sci. 94:6203-6215. https://doi.org/10.3168/jds.2011-4290.

Schukken, Y. H., M. J. Zurakowski, B. J. Rauch, B. Gross, L. L. Tikofsky, and F. L. Welcome. 2013. Noninferiority trial comparing a first-generation cephalosporin with a third-generation cephalosporin in the treatment of nonsevere clinical mastitis in dairy cows. J. Dairy Sci. 96:6763-6774. https://doi.org/10.3168/jds.2013-6713.

Searchable Proceedings of Animal Conferences. 2019. American Dairy Science Association. Accessed Feb. 9, 2020. https://spac.adsa.org/.

Sérieys, F., Y. Raguet, L. Goby, H. Schmidt, and G. Friton. 2005. Comparative efficacy of local and systemic antibiotic treatment in lactating cows with clinical mastitis. J. Dairy Sci. 88:93-99. https: //doi.org/10.3168/jds.S0022-0302(05)72666-7.

Shpigel, N. Y., D. Levin, M. Winkler, A. Saran, G. Ziv, and A. Bottner. 1997. Efficacy of cefquinome for treatment of cows with masti- 
tis experimentally induced using Escherichia coli. J. Dairy Sci. 80:318-323. https://doi.org/10.3168/jds.S0022-0302(97)75941-1.

Sol, J., O. C. Sampimon, H. W. Barkema, and Y. H. Schukken. 2000. Factors associated with cure after therapy of clinical mastitis caused by Staphylococcus aureus. J. Dairy Sci. 83:278-284. https:/ /doi.org/10.3168/jds.S0022-0302(00)74875-2.

Swinkels, J. M., P. Cox, Y. H. Schukken, and T. J. G. M. Lam. 2013a. Efficacy of extended cefquinome treatment of clinical Staphylococcus aureus mastitis. J. Dairy Sci. 96:4983-4992. https://doi.org/ 10.3168/jds.2012-6197.

Swinkels, J. M., V. Kromker, and T. J. G. M. Lam. 2014. Efficacy of standard vs. extended intramammary cefquinome treatment of clinical mastitis in cows with persistent high somatic cell counts. J. Dairy Res. 81:424-433. https://doi.org/10.1017/ S0022029914000442.

Swinkels, J. M., T. J. G. M. Lam, M. J. Green, and A. J. Bradley. 2013b. Effect of extended cefquinome treatment on clinical persistence or recurrence of environmental clinical mastitis. Vet. J. 197:682-687. https://doi.org/10.1016/j.tvjl.2013.03.010.

Tang, K. L., N. P. Caffrey, D. B. Nobrega, S. C. Cork, P. E. Ronksley, H. W. Barkema, A. J. Polachek, H. Ganshorn, N. Sharma, J. D Kellner, and W. A. Ghali. 2017. Restricting the use of antibiotics in food-producing animals and its associations with antibiotic resistance in food-producing animals and human beings: A systematic review and meta-analysis. Lancet Planet. Health 1:e316-e327. https://doi.org/10.1016/S2542-5196(17)30141-9.

Taponen, S., K. Dredge, B. Henriksson, A. M. Pyyhtia, L. Suojala, R. Junni, K. Heinonen, and S. Pyorala. 2003a. Efficacy of intramammary treatment with procaine penicillin $\mathrm{G}$ vs. procaine penicillin $\mathrm{G}$ plus neomycin in bovine clinical mastitis caused by penicillinsusceptible, gram-positive bacteria-a double blind field study. J. Vet. Pharmacol. Ther. 26:193-198. https://doi.org/10.1046/j.1365 $-2885.2003 .00473 . x$.

Taponen, S., A. Jantunen, E. Pyorala, and S. Pyorala. 2003b. Efficacy of targeted 5-day combined parenteral and intramammary treatment of clinical mastitis caused by penicillin-susceptible or penicillin-resistant Staphylococcus aureus. Acta Vet. Scand. 44:53-62. https://doi.org/10.1186/1751-0147-44-53.
Tomazi, T., T. A. F. Lopes, V. Masson, J. M. Swinkels, and M. V. Santos. 2018. Randomized noninferiority field trial evaluating cephapirin sodium for treatment of nonsevere clinical mastitis. J. Dairy Sci. 101:7334-7347. https://doi.org/10.3168/jds.2017-14002.

Tonin, F. S., I. Rotta, A. M. Mendes, and R. Pontarolo. 2017. Network meta-analysis: A technique to gather evidence from direct and indirect comparisons. Pharm. Pract. (Granada) 15:943. https://doi .org/10.18549/PharmPract.2017.01.943.

Truchetti, G., E. Bouchard, L. Descoteaux, D. Scholl, and J.-P. Roy. 2014. Efficacy of extended intramammary ceftiofur therapy against mild to moderate clinical mastitis in Holstein dairy cows: A randomized clinical trial. Can. J. Vet. Res. 78:31-37.

Turner, A., D. Tisdall, D. C. Barrett, S. Wood, A. Dowsey, and K. K. Reyher. 2018. Ceasing the use of the highest priority critically important antimicrobials does not adversely affect production, health or welfare parameters in dairy cows. Vet. Rec. 183:67. https://doi .org/10.1136/vr.104702

Vasquez, A. K. D. V. Nydam, M. B. Capel, B. Ceglowski, B. J. Rauch M. J. Thomas, L. Tikofsky, R. D. Watters, S. Zuidhof, and M. J. Zurakowski. 2016. Randomized noninferiority trial comparing 2 commercial intramammary antibiotics for the treatment of nonsevere clinical mastitis in dairy cows. J. Dairy Sci. 99:8267-8281. https://doi.org/10.3168/jds.2016-11258.

Wilson, D. J., P. M. Sears, R. N. Gonzalez, B. S. Smith, H. F. Schulte 3rd, G. J. Bennett, H. H. Das, and C. K. Johnson. 1996. Efficacy of florfenicol for treatment of clinical and subclinical bovine mastitis. Am. J. Vet. Res. 57:526-528.

World Health Organization. 2016. Critically important antimicrobials for human medicine - 5th Revision. WHO, Geneva. Accessed Feb. 07, 2020. https://www.who.int/foodsafety/publications/ antimicrobials-fifth/en/.

Wraight, M. D. 2003. A comparative efficacy trial between cefuroxime and cloxacillin as intramammary treatments for clinical mastitis in lactating cows on commercial dairy farms. N. Z. Vet. J. 51:26-32. https://doi.org/10.1080/00480169.2003.36326. 